\title{
Determining the time required to disinfect a sponge contaminated with Escherichia coli, using a commercial microwave
}

\author{
Amrita Bassan $^{1}$, Bobby Sidhu ${ }^{2}$, Ken Keilbart ${ }^{3}$, Fred Shaw $^{4}$ \\ 1 Lead Author, School of Health Sciences, British Columbia Institute of Technology, Burnaby, BC \\ 2 Supervisor, School of Health Sciences, British Columbia Institute of Technology, Burnaby, BC \\ 3 Contributor, BCIT Food Technology Lab, British Columbia Institute of Technology, Burnaby, BC \\ 4 Contributor, School of Health Sciences, British Columbia Institute of Technology, Burnaby, BC
}

\begin{abstract}
Background: Disinfection and sanitation are important in areas where food is involved. Thorough cleaning is a necessity to prevent growth of harmful pathogens that could affect human health. Sponges used for cleaning can serve as a vehicle for cross-contamination on food preparation surfaces. There are various methods that could be used to disinfect contaminated sponges. The usage of a microwave is one suggested method.

Objective: The purpose of this study was to determine if the Scotch-Brite ${ }^{\mathrm{TM}}$ Brand, cellulose sponges contaminated with E.coli $\left(10^{5} \mathrm{cfu} / \mathrm{ml}\right)$ could be disinfected using a microwave set at three timings ( 30 seconds, 1 minute, and 2 minutes).

Methods: The Hygiena MicroSnap was used to detect the presence (or absence) of E.coli in sponges after microwave heating. The relative light units (RLU) indicated in the monitor determined whether there were any remaining coliforms in the sample after microwaving.

Results: Statistical analysis was conducted using Microsoft Excel and NCSS. After heating sponges for 30 seconds, $100 \%$ of the samples detected no E.coli. After heating for 1 minute, $70 \%$ of the samples had no E.coli present. After heating for 2 minutes, $100 \%$ of the samples detected no E.coli. The p-value of 0.03567 concludes that the results were statistically significant at the $5 \%$ significance level.

Discussion: The results of this study indicate that sponges contaminated with E.coli can be disinfected using microwave heating. EHOs, food establishment operators, and the general public can use this knowledge to re-use their old sponges and avoid further cross-contamination.

Conclusions: The results indicate that microwave time is associated with the presence or absence of E.coli in a sponge. However, E.coli was present in 3 samples microwaved at 1 minute. This suggests further studies are required to confirm the findings of this study. In addition, further studies are required to determine what specific time is sufficient to completely eliminate the E.coli in a contaminated sponge.
\end{abstract}

Keywords: E.coli, sponge, disinfection, microwave, MicroSnap, cross-contamination, EHO.

\section{INTRODUCTION}

A microwave oven is an appliance that is used for cooking food, reheating cooked foods and heating liquids. It uses electromagnetic waves, called microwaves, to generate thermal energy. When electromagnetic energy comes in contact with the food, water molecules in the food rotate (Canadian Centre for Occupational Health and Safety [CCOHS], 2013). This movement allows the temperature to rise; hence causing the food to heat. Temperature in a microwave can rise up to $100^{\circ} \mathrm{C}$ (Vollmer, 2004). According to the CCOHS (2013), most conventional microwaves generate $2450 \mathrm{MHz}$ of energy. With high microwave frequencies, and the ability to heat to high temperatures, the microwave is an appliance that can be considered for additional uses. One such use is disinfection.

Disinfection and sanitation are necessary to reduce harmful pathogens that could affect human health. In a food premises, a three-compartment sink system is the ideal setup for washing dishes. The three sinks (used to wash, rinse, and sanitize) maximize the cleanliness and decrease the amount of contamination of equipment (Toronto Public Health, 2004). Sponges are also a part of this cleaning process as they aid in removing debris and cleanse dishes mechanically. They are certainly a part of the washing cycle, and are also used to clean surfaces. Ideally, the effective way to clean surfaces is using a bucket filled with warm water and soap, followed by a spray bottle of sanitizer. Some individuals may choose not to use 
disinfectants for aesthetic reasons, such as odor or sensitivity. Furthermore, some may be hesitant to use sanitizers because they are known to be harsh on hands, equipment, and hazardous for children and pets. A simpler and quick method of disinfection of sponges must be considered for those who are uncomfortable using sanitizers, and for those who may not be familiar with other methods.

As a student in Environmental Health at the British Columbia Institute of Technology (BCIT), the author was exposed to various methods of disinfection that operators, and even homeowners could apply. One of the instructors mentioned how a microwave could be used to disinfect rags. Another instructor suggested the same subject as a research topic. With the support and guidance of the instructors, and personal interest, the author conducted a study on microwaving sponges for the purpose of disinfection.

\section{Proposed Project}

The proposed research topic was to determine at what time would a flat, rectangular kitchen sponge be disinfected when heated in a microwave. The presence or absence of an indicator organism (Escherichia coli) was examined after the sponge had been microwaved at various times (30 seconds, 1 minute, or 2 minutes).

\section{Rationale for the Study}

Kitchen sponges are frequently used throughout the day. Multiple uses, without proper disinfection, can lead to harborage of microorganisms. When these sponges come into contact with food preparation surfaces, there is potential for a serious illness to occur. Several studies have indicated that sponges serve as a vehicle for cross-contamination of food preparation surfaces (Hilton \& Austin, 2000; Lee, 2006, Sharma, Eastridge \& Mudd, 2009). It is not common knowledge whether a microwave can be used to disinfect dirty kitchen sponges. This study provides information to the general public whether a microwave can be used as an easy and simple way to disinfect their contaminated sponges.

\section{LITERATURE REVIEW}

\section{Legislation}

Although there is no specific legislation covering kitchen sponges, there is reference to equipment used on food contact surfaces. Furthermore, there is no legislation suggesting a microwave can be used as a method of disinfection; however, there are considerations for the construction and use of a microwave.

Food Premises Regulation (1999) - This regulation falls under the British Columbia Public Health Act. According to Section 17, any equipment that comes in contact with food or operated on the food premises must be "maintained in a sanitary condition" and "washed and sanitized in a manner that removes contamination." This suggests a cleaning sponge would fall under equipment used on food contact surfaces; hence, must be cleaned in a sanitary manner.

Radiation Emitting Devices Regulations (2006) - This regulation falls under Health Canada's Radiation Emitting Devices Act. According to Schedule 2 - Part 3 , a commercial microwave is used for a commercial establishment, an industrial establishment, or in or with a vending machine. The regulation outlines design and construction specifications to operate in Canada, but does not specify that it can be used as a disinfection method.

\section{Contamination of Surfaces}

Pathogenic bacteria growth occurs due to a combination of factors. These factors include: temperature, protein, available water, $\mathrm{pH}$, oxygen and time. Growth of pathogens may lead to a foodborne illness, which is caused by the consumption of contaminated food or water. If a contaminated sponge or a rag is used on a surface or equipment handling food, pathogens can cause crosscontamination, potentially leading to a foodborne illness.

The kitchen is found to be an ideal place, or hotspot, for the harborage of pathogens (Donofrio et al., 2012). One study found that the kitchen had the highest heterotrophic plate count when compared to the bathroom, personal items and pet items (Donofrio et al., 2012). Improper sanitation in the kitchen has a greater potential for a foodborne illness to occur when compared to other areas of the household (Hilton \& Austin, 2000; Lee, 2010; Donofrio et al., 2012). This shows that practicing proper sanitation is crucial to decreasing foodborne illness transmission in the kitchen. Sponges can provide a route of crosscontamination for a pathogen, leading to a foodborne illness (Hilton \& Austin, 2000). These sponges get contaminated while being used to wipe surfaces. Based on the conditions the sponges are usually left in, the chances for pathogen survival increases within the moist conditions (Park, Bitton \& Melker, 2006). 
Sponges are commonly used to clean up spills, grab hot handles, and wipe down equipment and counter tops. Those that are fresh and clean are prone to accumulate microorganisms as they are used. If not disinfected adequately, re-use of soiled sponges can contaminate the surfaces they are used to wipe. For instance, if a sponge with a high bacterial load is used to clean a cutting board, any food placed on that cutting board would pick up the bacteria, leading to a cross-contamination. One study examined the survival of microbes, Salmonella enteritidis, $S$. aureus, and Campylobacter jejuni, on stainless steel surfaces (Kusumaningrum et al. 2003). Part of the study looked at how sponges transfer these microbes onto the stainless steel surfaces. It was found that 21$43 \%$ of the microbes were transferred to the surfaces, while $50 \%$ still remained in the sponges. According to the United States Food and Drug Administration (2013), the use of sponges is prohibited on food contact surfaces that have been cleaned, sanitized or are in-use. In other words, sponges should only be used before rinsing and sanitizing equipment, rather than to clean food contact surfaces.

\section{Kitchen Sponge vs. Rag}

Is there a difference in using a sponge versus a rag? Sponges and rags must be cleaned daily, replaced frequently and stored in a dry location ("Kitchen Sponge Safety," n. d.). Spills should be cleaned up using a paper towel or disinfectant wipes, rather than use sponges on countertops ("Kitchen Sponge Safety," n. d.). Several studies show that the sponge is able to hold more microbes than a rag, however the rag is able to spread microbes onto a surface quicker than a sponge (Donofrio et al., 2012; Hilton \& Austin, 2000; Kusumaningrum, 2003; Lee, 2006). In one such study, Hilton and Austin (2000) tested for the presence of Salmonella, Campylobacter and Staphylococcus aureus in kitchen cloths and sponges. It was found that total viable counts of $S$. aureus were higher on sponges than on cloths. Furthermore, a cloth transferred more organisms onto a cutting board than a sponge. This suggests the different physical characteristics of a sponge and cloth can impact the amount of microorganisms ending up on food contact surfaces (Hilton \& Austin, 2000; Donofrio et al., 2012). A cloth has a larger surface area, allowing easier transfer of microbial load. A sponge would be able to harbor more microbes than a cloth because of its structure and lesser surface area compared to a rag.

Another study looked at the effect of ultraviolet (UV) sterilization, with or without heat $\left(50^{\circ} \mathrm{C}\right)$, on sponges and dishcloths after they had been contaminated with
Escherichia coli (Lee, 2006). It was found that different types/materials of sponges and dishcloths influence the survival and growth of E.coli. Lee (2006) says, "microbial cells contained on commercial sponges/cloths grow quickly depending on the materials they are made from (p. 721)." Rayon, cotton and polyester materials was shown to harbour higher levels of E.coli growth (approximately 7.5-7.6 log colony forming units (CFU)/each) before having any treatment. Rayon and cotton samples had the most significant reduction (1.8 and $3.0 \log$ CFU, respectively) after the combined treatment of heat and UV (Lee, 2006).

Based on the comparisons between sponges and rags, the author chose to use sponges as the basis of the study. This is because the author tends to wash rags in the washing machine and discards sponges without a method of disinfection. The author wants to determine if sponges can be reused if they are sufficiently disinfected using a microwave.

\section{Using a Microwave for Sterilization}

Earlier studies indicate that only leaving a kitchen sponge to dry is insufficient to inactivate pathogens (Lee, 2010; Park, Bitton \& Melker, 2006). Another method or an additional step is required to sterilize contaminated sponges. One study looked into the use of microwave radiation as a sterilization method. Polyester and cotton cloths were contaminated with test organisms: S. aureus, E. coli and Bacillus cereus, and it was examined at what times these materials no longer indicated the organisms (Reagan, Rolow \& Urban, 1982). Samples for each fabric were exposed to $0,1,3,5$ or 7 minutes in the microwave. The results exhibited that with increasing microwave radiation exposure, the levels of bacteria decreased. E.coli was found to be the most sensitive to the microwave radiation. $91.54 \%$ E.coli was reduced within $1 \mathrm{~min}$, and $100 \%$ by $3 \mathrm{~min}$ (Reagan, Rolow \& Urban, 1982). Furthermore, there was no significant difference with the type of fabric the cloth was. This study showed that microwaving cloths was able to kill test organisms as the microwave time increased. The author proposes to do a similar study using sponges. The author wants to study if the indicator organism, E.coli, is killed in sponges as the microwave time increases.

Another study done by Park, Bitton and Melker (2006) showed that using a microwave could significantly decrease microbial load and inactivate pathogens on sponges. 30 seconds of exposure time in the microwave led to $100 \%$ inactivation of E.coli in a kitchen sponge (Park, Bitton \& Melker, 2006). 
These sponges were inoculated with raw wastewater before any exposure to microwave radiation. Furthermore, $99 \%$ of the total bacterial count was reduced within 2 minutes. The surface temperature (range of $79.4^{\circ} \mathrm{C}$ at 1 minute to $80.8^{\circ} \mathrm{C}$ at 4 minutes), and internal temperature (range of $91.4^{\circ} \mathrm{C}$ at 1 minute to $90.4^{\circ} \mathrm{C}$ at 4 minutes) of the sponge were also recorded (Park, Bitton \& Melker, 2006). This study indicates that 30 seconds of microwave time could kill $100 \%$ of E.coli in a sponge contaminated with raw wastewater. The author wanted to confirm these findings with E.coli as the test organism used to contaminate the sponges.

Lee (2010) found that "the combination of UV and heat $(50 \mathrm{C})$ was the most effective in reducing levels of E.coli" (p. 727). This study also shows that heat influences the effectiveness of disinfection, depending on the material of a sponge and dishcloth. Furthermore, Sharma, Eastridge and Mudd (2009) found that using the microwave and dishwasher significantly reduced microbial load on kitchen sponges more than chemical treatment. In this study, sponges were inoculated in ground beef slurry, and then microwaved for 1 minute. Less than $0.410 g$ $\mathrm{CFU} /$ sponge of aerobic bacteria survived (Sharma et al., 2009). To compare, control sponges that were received no disinfection treatment had 7.5 CFU/sponge. This study showed that application of heat influences the destruction of E.coli. Heat generated by a microwave would potentially inactivate the microbes.

\section{Precaution of Placing Sponge in Microwave}

BBC News (2007) reported that a fire broke out in a microwave when a person in Shropshire, England used a microwave to disinfect a dishcloth. The local fire department advised that sponges and dishcloths should not be microwaved because the power of a microwave and moisture in a sponge or cloth varies. A dry sponge or rag should not be heated in a microwave. To prevent a fire hazard, the sponge and rag should be wet and contain no metal (Park, Bitton \& Melker, 2006; BBC News, 2007). Furthermore, the items must be handled with care when removing them after heating, to prevent burns from heat and steam.

\section{Role of an Environmental Health Officer}

The role of an Environmental Health Officer (EHO) regarding this study involves education. The hazards of spreading illness through a sponge may not come across everyone's minds. Perhaps people do not discard their sponges until a very long time of usage.
This paper would help EHOs provide knowledge and education of an alternative method to disinfecting sponges.

\section{Concentration of Escherichia coli}

E.coli is gram negative, facultative anaerobe. Some serotypes are pathogenic which can cause a foodborne illness. Contaminated food or water can serve as a vehicle of transmission, leading to an illness such as: "diarrhea, urinary tract infections, respiratory illness, bloodstream infections, and other illnesses" (CDC, 2013). Various studies mentioned in this paper used E.coli as one of their test organisms to inoculate sponges. To remain consistent with the studies mentioned in this paper, the author chose to use E.coli as the test organism for the study.

\section{PURPOSE OF STUDY AND RESEARCH QUESTION}

The purpose of the study is to evaluate the presence or absence of E.coli on kitchen sponges after heating them at set times using a commercial microwave. Also, to determine the correct time of heating in a commercial microwave where E.coli would have total inactivation.

\section{Null Hypothesis}

There is no association between the presence (or absence) of E.coli in sponges and microwaving time (30 seconds, 1 minute, or 2 minutes).

\section{Alternative Hypothesis}

There is an association between the presence (or absence) of E.coli in sponges and microwaving time (30 seconds, 1 minute, or 2 minutes).

\section{MATERIALS AND METHODS}

\section{Materials}

All materials were handled aseptically. Wherever required, a Bunsen burner was used to sterilize tips of equipment (i.e. test tubes). The lab setup was cleaned before and after the procedure. Refer to Table 1 for an outline of the materials that were used in this study. 
Table 1: Materials Used for the Study

\begin{tabular}{|c|c|}
\hline Materials & Used For \\
\hline Nitrile gloves & To handle all materials aseptically. \\
\hline Scotch-Brite Sponges & The subject of study. \\
\hline Ruler & To measure the sponge size. \\
\hline Scissors & To cut the sponges into small squares (1.5” x $1.5 "$ x $5 / 8$ "). \\
\hline Escherichia coli & Bacteria used to inoculate sponge pieces. \\
\hline Tryptic Soy Agar Plate & Streaked with E.coli and incubated to allow bacterial growth. \\
\hline Lauryl Tryptose Powder & Powder used to prepare the broth for the E.coli inoculum. \\
\hline Peptone Powder & Powder used to prepare the solution for the dilutions. \\
\hline Distilled Water & To make a peptone solution using the peptone powder. \\
\hline Peptone Solution & For dilution series to obtain the desired starting E.coli concentration. \\
\hline Test Tubes & Storage of E.coli broth after serial dilutions. \\
\hline Tongs 1 & To place a sponge piece into a Ziploc bag. \\
\hline Pipette & To transfer E.coli broth to Ziploc bag containing a sponge piece. \\
\hline Ziploc Bags & To soak sponge pieces in E.coli broth. \\
\hline Small Plastic Container & $\begin{array}{l}\text { The inoculated sponge piece will be placed into the container. The container } \\
\text { will then placed into microwave for heating. }\end{array}$ \\
\hline $\begin{array}{l}\text { Microwave } \\
\text { Brand: Oster } \\
\text { Model: OGB7801 }\end{array}$ & Used to heat the sponges at specific times. \\
\hline Tongs 2 & To pick up heated sponge piece and place into stomacher bag. \\
\hline Stomacher & To squeeze liquid out of sponge to be used for the MicroSnap. \\
\hline $\begin{array}{l}\text { Hygiena MicroSnap } \\
\text { Enrichment Swab }\end{array}$ & $\begin{array}{l}\text { Contains a specific growth medium for the specific microbial detection } \\
\text { (Hygiena, 2013a). }\end{array}$ \\
\hline Incubator & To incubate the MicroSnap swabs at $37^{\circ} \mathrm{C}$. \\
\hline $\begin{array}{l}\text { Hygiena MicroSnap Detection } \\
\text { Swab }\end{array}$ & $\begin{array}{l}\text { Contains a bioluminogenic substrate for the specific microbial detection } \\
\text { (Hygiena, 2013a). }\end{array}$ \\
\hline Hygiena Monitor & To detect presence or absence of E.coli in sponges after microwaving. \\
\hline $\begin{array}{l}\text { Office Supplies (pen, paper, } \\
\text { ruler) }\end{array}$ & To make tables and record results at the lab. \\
\hline Computer & To analyze results using Microsoft Excel and NCSS software. \\
\hline
\end{tabular}

\section{Standard Methods}

The Food Technology Laboratory located at BCIT was used to carry out the study. The author wore nitrile gloves throughout the entire procedure to avoid any chances of contamination, and to ensure the study was performed in a sterile manner.

A fresh, sterile tryptic soy agar plate was streaked with E.coli and incubated for 24 hours, allowing the bacteria to multiply and grow (K. Keilbart, personal communication, November 7, 2013). A lauryl tryptose broth (LTB) was also prepared using $35.6 \mathrm{~g}$ of the lauryl tryptose powder in 1L of distilled water (Himedia, 2011). The broth was inoculated using the E.coli $\left(10^{9} \mathrm{cfu} / \mathrm{ml}\right)$ grown on the agar plate and then placed in a shaking water bath.
A peptone solution $(1 \mathrm{~g} / \mathrm{L})$ was prepared for diluting the E.coli from $10^{9} \mathrm{cfu} / \mathrm{ml}$ to $10^{5} \mathrm{cfu} / \mathrm{ml}$. Each test tube had $9 \mathrm{ml}$ of peptone, which was used for the dilutions of E.coli.

Scotch-Brite ${ }^{\mathrm{TM}}$ Brand, Cellulose Sponge Handy Packs were purchased for the study (Scotch-Brite Products, 2013a). Each sponge was cut into smaller pieces ( $1.5 \times 1.5 \times 5 / 8$ inches) with scissors.

Using sterile tongs, each sponge piece was inserted into a Ziploc bag to be soaked with the E.coli broth for 24 hours.

After soaking with E.coli, the sponges were transferred to a container using tongs, and microwaved individually. Before placing them into 
the microwave each sponge sample was soaked with $5 \mathrm{ml}$ of tap water so it would not dry out and cause a fire hazard. The Oster microwave (Model: OGB7801) was used to heat one sponge piece at a time. Three heating times were used (30 seconds, 1 minute and 2 minutes) where each sample was heated once. A total of 10 samples were used per timing.

After each heating, the sponge sample was cooled to room temperature. Using tongs, the sponge was transferred into a stomacher bag and then placed into the stomacher. The stomacher squeezed any remaining excess liquid from the sponge. This liquid $(1 \mathrm{~mL})$ was collected for the MicroSnap enrichment.

The MicroSnap was used to detect the presence or absence of E.coli in each sponge sample. The device uses a two-part system: enrichment and detection (Hygiena, 2013a). 1mL of the collected sample liquid was pipetted into the enrichment swab (HygienaTV, 2011). The swab has an attached specific growth medium that was snapped and added into the pipette. The enrichment swab was incubated for 8 hours at $37^{\circ} \mathrm{C}$ (HygienaTV, 2011). After incubation, 100uL (3 drops) of liquid from the enrichment tube was added to the detection swab (Hygiena, 2013a). The detection swab has an attached bioluminogenic substrate that was snapped and added into the pipette. The detection swab was incubated for 10 minutes at $37^{\circ} \mathrm{C}$ (HygienaTV, 2011). After incubation, the detection swab was inserted into the Hygiena monitor for a reading. The luminometer in the monitor output whether E.coli was present or absent in the sample based on the relative light units (RLU) (Hygiena, 2013b). Table 2 outlines the presence/absence RLU threshold values for the Hygenia Monitor.

\section{Table 2: Presence/absence Threshold Values}

\begin{tabular}{cc}
\hline Result & Hygenia Monitor (systemSURE II) \\
\hline Absent & 0 \\
Caution & 1 \\
Present & $\geq 2$ \\
\hline
\end{tabular}

(Hygiena, 2013c)

Raw data was recorded and were entered into Microsoft Excel for analysis (Microsoft Corporation, 2011a). The NCSS (2012) software was used to determine if the null hypothesis would be rejected or not.

\section{Reliability and Validity of Measures}

Reliability. The MicroSnap has received Performance Tested Method Validation from the AOAC Research

Institute (Hygiena, 2013d). The test must be carried out in a specific procedure in order for the MicroSnap to work properly. In other words, the MicroSnap is administered the same way each time. Although the MicroSnap has not been used at BCIT before, it's main feature, the luminometer has been use in other devices, such as to measure adenosine triphosphate (ATP).

Validity. The MicroSnap will have a field calibration using a calibration check according to the specs of the manual (F. Shaw, personal communication, November 7, 2013). It is a product of EnSURE, which is used for other systems such as: Surface ATP, Water ATP, Allergen Prevention, Alkaline Phosphatase, and Acid Phosphatase (Hygiena, 2013a). The researcher will be using the MicroSnap during the pilot study, and will be able to provide feedback whether the instrument is effective or not.

\section{Calibration of Instruments}

The MicroSnap had a field calibration check as per specs of the manual. All other materials did not require calibration because they were equipment, not instruments.

\section{Inclusion and Exclusion Criteria}

Table 3 outlines the criteria for inclusion and exclusion for this study. Specific Scotch-Brite sponges were used for this study. All other sponges were excluded.

\section{Ethical Considerations}

Ethical considerations were not applicable to this study because it did not require a survey and no humans were involved (H. Heacock, personal communication, November 5, 2013).

Table 3: Criteria for Inclusion and Exclusion

\begin{tabular}{|c|c|}
\hline Inclusion & Exclusion \\
\hline $\begin{array}{l}\text { Scotch-Brite }{ }^{\mathrm{TM}} \text { Brand, Cellulose Sponge Handy Pack } \\
\text { (Scotch-Brite Products, 2013a). }\end{array}$ & $\begin{array}{l}\text { All other sponges, such as: } \\
\text { - Scrubs; }\end{array}$ \\
\hline $\begin{array}{l}\text { The pack provides } 4 \text { sponges. Each sponge was cut } \\
\text { into small pieces }(1.5 \times 1.5 \times 5 / 8 \text { inches }) \text {. }\end{array}$ & $\begin{array}{l}\text { - Soap dispensing dishwands; } \\
\text { - Green clean sponges, and } \\
\text { - Scouring pad (Scotch-Brite Products, 2013b) }\end{array}$ \\
\hline
\end{tabular}




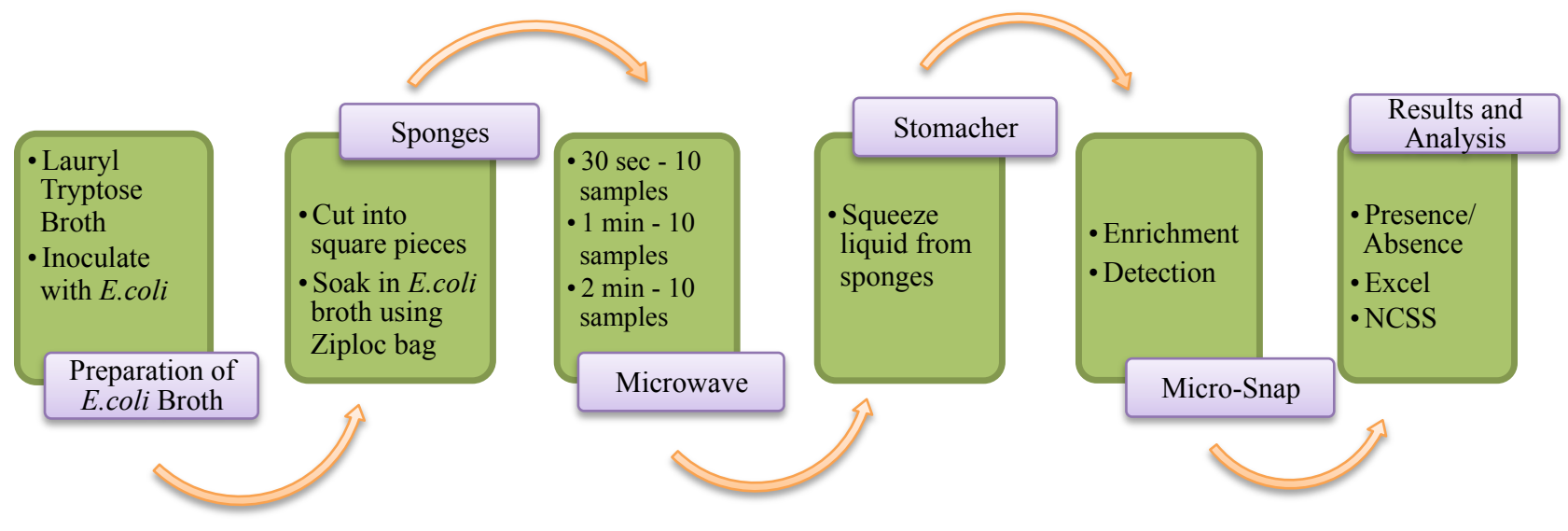

\section{Pilot Study}

Part 1. To determine the concentration of E.coli that was used for the actual study. Ken Keilbart, from the BCIT Food Technology Lab, suggested that the concentration of E.coli used would have an impact on the study (personal communication, November 7, 2013). Furthermore, this part of the study would determine what the ideal concentration is on surfaces. E.coli, from a tryptic soy agar plate, was used to inoculate the lauryl tryptose broth. The broth was then incubated, allowing the bacteria to grow to $10^{9}$ $\mathrm{cfu} / \mathrm{ml}$ (K. Keilbart, personal communication, November 7, 2013). The broth underwent serial dilutions using distilled water to obtain a concentration of $10^{5} \mathrm{cfu} / \mathrm{ml}$.

Part 2. Refer to Figure 1 for the study outline. Due to time constraints and access to the MicroSnap reagents, the pilot study planned for December 2013, was incomplete. $5 \mathrm{ml}$ tap water was added to each sponge before microwaving in order to prevent the sponge from drying out, and to prevent a fire hazard from microwaving a dry sponge (K. Keilbart, personal communication, December 16, 2013). The study was completed in January 2014. Both positive (E.coli at $10^{5} \mathrm{cfu} / \mathrm{ml}$ ) and negative (peptone only) controls were used. In addition, due to the expense of E.coli detection tubes, total coliform swabs were used for detection instead. The total coliform swabs detected E.coli as well. Since the only contaminant present in the sponges was E.coli, total coliform swabs worked just as well. If there were any detection of total coliforms, only then specific E.coli detection swabs would be used (F. Shaw, personal communication, January 7, 2013). Otherwise, the total coliform detection swabs represented the results for presence or absence.

\section{RESULTS}

\section{Description of Data}

The author collected dichotomous, nominal data. The data was nominal because it categorized if E.coli in a sponge is present or absent after being heated in the microwave. Since there only were two options for the results, the data was dichotomous. The nominal data would be represented as counts, percentages and proportions of the total (Heacock, Crozier, \& Sidhu, 2012a). Results for presence/absence was determined by threshold values as described in the directions for the use of MicroSnap.

\section{Descriptive Statistics}

Figures 2 outlines the results for the study. 10 sponges $(100 \%)$ had absence of E.coli after being microwaved for 30 seconds (Table 4). 7 sponges (70\%) had absence of E.coli after being microwaved for 1 minute (Table 4). 10 samples contained no E.coli after being microwaved for 2 minutes (Table 4). Microsoft Excel (Microsoft Corporation, 2011a) and Microsoft Word (Microsoft Corporation, 2011b) were used to generate these statistics. The NCSS (2012) software was used to run a chi-square analysis.

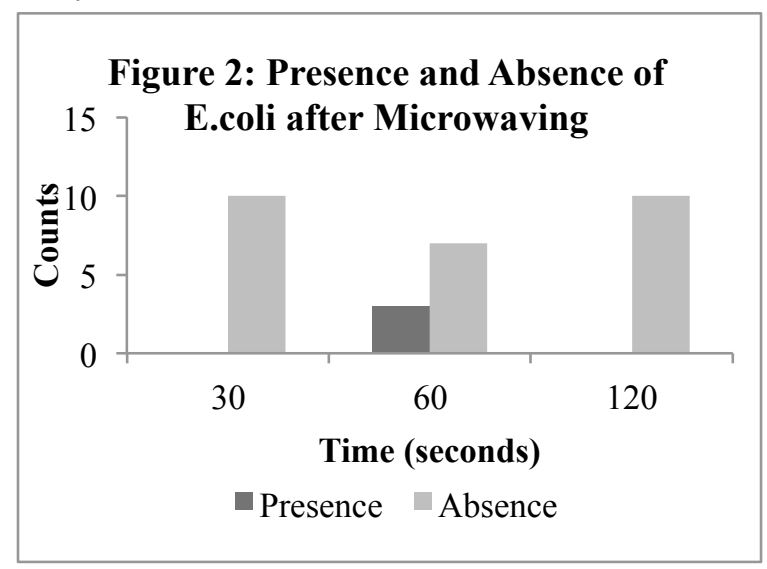


Table 4: E.coli Presence and Absence

\begin{tabular}{|c|c|c|c|c|}
\hline & \multicolumn{2}{|c|}{ Present } & \multicolumn{2}{c|}{ Absent } \\
\hline Time (seconds) & Count & Percentage & Count & Percentage \\
\hline $\mathbf{3 0}$ & 0 & $0 \%$ & 10 & $100 \%$ \\
\hline $\mathbf{6 0}$ & 3 & $30 \%$ & 7 & $70 \%$ \\
\hline $\mathbf{1 2 0}$ & 0 & $0 \%$ & 10 & $100 \%$ \\
\hline
\end{tabular}

\section{Inferential Statistics}

The author conducted a chi-square analysis since the data represented nominal values. Chi-square tests are non-parametric, robust, and simple to calculate and they allow flexible manipulation of the data (McHugh, 2013; Heacock, Crozier, \& Sidhu, 2012b).

\section{Statistical Packages Used}

The statistical packages used for the analysis of this study consisted of Microsoft Excel, NCSS, and Microsoft Word. Raw data was entered into Microsoft Excel to generate graphs and charts for the results. A Chi-square analysis was conducted using NCSS. The p-value was also determined using NCSS. Microsoft Word was used to organize the results.

\section{Results of Statistical Analysis}

Null Hypothesis (Ho). There is no association between the presence (or absence) of E.coli in sponges and microwaving time (30,60, 120 seconds).

Alternate Hypothesis ( $\mathrm{Ha}$ ). There is an association between the presence (or absence) of E.coli in sponges and microwaving time (30, 60, 120 seconds).

Interpretation of Results. Using the chi-square analysis performed by NCSS, the author was able to interpret the results of the study. 10 samples were microwaved at 30,60, and 120 seconds, respectively. This gives a total of 30 samples for the entire study. The p-value was found to be 0.03567 ; therefore, the author rejected the null hypothesis. At the 5\% significance level, it was concluded that there is an association between the presence (or absence) of E.coli in a sponge and time the sponge was microwaved.

Alpha Error. An alpha error occurs when the test results indicate there is an association between two groups (i.e. reject $\mathrm{Ho}$ ), but in reality, there is no association (i.e. do not reject Ho) (Heacock, Crozier, \& Sidhu, 2012a). In this study, the results indicate the $\mathrm{p}$-value is within the acceptable alpha value ( $\mathrm{p}<$ $0.05)$. The p-value of 0.03567 is significant at the $5 \%$ level but not at the $1 \%$. To minimize alpha errors, the acceptable p-value would need to be decreased. This indicates that there is a possible alpha error since the $\mathrm{p}$-value is near the borderline significance.

\section{DISCUSSION}

The author conducted this study to test E.coli survival after microwave treatment. It was found that after microwaving for 30 and 120 seconds, there was $100 \%$ inactivation of E.coli on the sponge samples. After 60 seconds of microwaving, 30\% of the samples had pathogen survival. Although 3 samples detected E.coli after 60 seconds microwaving, the pvalue of 0.03567 was significant at the $5 \%$ level. Hence, the author rejected the null hypothesis and concluded that there was an association between pathogen survival and microwave time. The reason for pathogen survival after 60 seconds of microwaving, yet no survival at 30 seconds indicates a possible alpha error as described in the results. The results of this study are in agreement with previous studies indicating that pathogen survival decreases with heat treatment and a microwave could be used as a technique to sterlize.

\section{Relation to Previous Studies}

Based on the results of this study, the author concluded that there is a relationship between E.coli survival on sponges and microwave time. Background information from BCIT and previous studies were used to build a basis for this study. Sponges are commonly found in kitchens, and could serve as a vehicle for cross-contamination. Donofrio et al. (2012) discussed how kitchens are ideal hotspots for harborage of pathogens. Consequently, cross-contamination from sponges in a kitchen could lead to a foodborne illness. This indicates that along with cleaning and sanitation of the main kitchen equipment, surfaces and items, sponges need to be given some attention for their disinfection. One study found that $50 \%$ of microbes still remained on sponges after wiping them on stainless steel surfaces (Kusumaningrum et al., 2003). Half of the microbes remaining on sponges raises a concern for food 
safety. This is because with a large amount of microbes remaining on sponges, and with multiple use of the sponge, the number of microbes being transferred to food contact surfaces also increases. The author researched if a microwave could be used to disinfect sponges contaminated with E.coli. Microwave heat and time were two important factors for this experiment that had an impact on E.coli survival.

According to Lee (2006), the material has an impact on the growth and survival of E.coli on a sponge. The author decided to use only one type of sponge brand, Scotch-Brite, which shows the results of this study are limited to that single sponge type and brand. This is further discussed in the limitations section of this paper. In addition, Lee (2006) found that a combined treatment of heat and UV decreased the CFUs compared to UV treatment alone. The author agrees heat has an impact on the pathogen survival. The microwave heat had an association with the E.coli survival as shown in the results of this study.

The author confirmed the findings of Park, Bitton and Melker (2006). Their study looked at sponges contaminated with raw wastewater, and found $100 \%$ of E.coli was inactivated after 30 seconds of microwaving. Although the author used E.coli as the only pathogen on the sponges, there was still $100 \%$ inactivation after 30 seconds of microwaving. In addition, the author confirmed findings of Reagan, Rolow and Urban (1982) that a microwave could be used as a sterilization method. Reagan, Rolow and Urban (1982) had used cloths, whereas the author for this study used sponges. The results Reagan, Rolow and Urban (1982) and the study in this paper indicated that heat from the microwave was associated with the inactivation of pathogens.

Although the author confirmed that 30 seconds of microwave time inactivated $100 \%$ of E.coli in the sponges, it could not be determined what specific time was adequate to eliminate all the E.coli. The results indicate 3 sponge samples were positive for E.coli after 1 minute of microwaving. Presence at the 1-minute mark indicated a caution result. The MicroSnap detection had a value of 1 for 3 samples, which shows a caution result. This means the 1minute mark may not have been adequate for total inactivation of E.coli in those samples. Hence, further studies would be required to confirm the findings of this study.

\section{Impact on Public Health Practices}

Previous studies have shown that a sponge is able to hold more microbes than a rag. Hilton and Austin (2000) found there were higher counts of S. aureus on sponges than on kitchen cloths. This shows pathogen accumulation is found more on sponges than on cloths. Also, the US Food and Drug Administration (2013) indicate a sponge should not be used on food contact surfaces. This is to prevent cross-contamination as sponges can harbor various pathogens. Nonetheless, if sponges can be disinfected using a microwave, a sponge then could essentially be used on food contact surfaces. The US Food and Drug Administration could change their policy and allow sponges to be used on food contact surfaces as long as they have been disinfected appropriately. Other policies and legislation could use the results from the study discussed in this paper. The Food Premises Regulation (1999) could add sponges as a type of equipment. The Radiation Emitting Devices Regulation (2006) can add that a microwave can also be used as a disinfection appliance for sponges. Most importantly, those who prefer not to discard their contaminated sponges often can use this study and results as a substitute in reusing the sponges, as long as they are disinfected.

The results of this study were based on a new detection method, the MicroSnap. The author would be able to provide feedback to BCIT about how efficient and reliable the MicroSnap method was. Indeed, the author found the MicroSnap method was easy to use, and the readings were straightforward to interpret. The MicroSnap should be able to output the same results as traditional methods, such as plating. EHOs would be able to educate operators on another way to sanitize sponges used in a food premises establishment. Importantly, whoever is disinfecting the sponges must ensure the sponge is moist. A dry sponge being microwaved is a fire hazard. This method is useful for those who do not like using chemicals to sanitize.

\section{RECOMMENDATIONS}

Based on this study, microwaving sponges could be used as another method of disinfection. This method can be of assistance in both the household and in food service establishments. To prevent a fire hazard, the sponge must be moist prior to microwaving. Finally, EHOs can use this study to educate operators and their staff about disinfecting the sponges they use. 
EHOs would also ensure the operator knows a dry sponge should not be microwaved.

\section{LIMITATIONS}

The author was able to only measure 10 samples for 3 different times due to budget constraints. The budget constraints had an impact on the sample size, which in turn could have decreased the reliability and validity of the data. Furthermore, time constraints due to the author's schedule limited the amount of testing that could have been conducted. With an increase of budget and time, the author would have been able to test more samples.

There were also some limitations when the study was being conducted. The incorrect media for the MicroSnap Detection was ordered for the pilot study. This delayed the pilot study completion. Also, there were two other researchers using the E.coli broth (before dilutions), which could have increased the chance of contamination. In order to avoid these limitations, the correct media should have been used for the pilot study, and each researcher should have used their own set of E.coli broth.

In addition, the specificity of the sponge limited the study. The flat sponge was a particular brand and

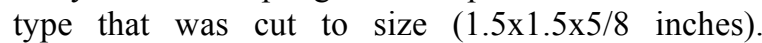
Perhaps using different styles and sizes of the sponge would enhance the study. This is further outlined in the suggestions for Future Research section below.

\section{FUTURE RESEARCH}

The following are suggestions for future studies involving the experiment presented in this study:

- Use a bigger sample size for each microwave time;

- Use different types of sponges (i.e. round, square, scrubs, dish wands, scouring pads, etc.);

- Use different sizes of sponges (i.e. full size as opposed to cut portions);

- Use different brands of sponges;

- Set microwave times lower than 30 seconds;

- Use a different concentration of E.coli;

- Repeat the experiment using traditional methods;

- Repeat the experiment using dish cloths;

- Repeat the experiment using a different pathogen;

- Repeat the experiment using more than one pathogen.

\section{CONCLUSIONS}

E.coli inoculated on sponge samples were impacted by microwave heating. $100 \%$ of the sponge samples that were microwaved at 30 and 120 seconds completely destroyed the E.coli. This indicates that the survival of the E.coli pathogen in a sponge is associated with microwave time. Although the author confirmed with previous studies that 30 seconds of microwave time had total inactivation of E.coli in the sponges, it could not be determined what time would be adequate to kill all the E.coli in the sample. Due to the presence of E.coli in three samples at the 60second mark, further studies are necessary to confirm the findings for this study. Lastly, future studies could research the specific microwave time for total inactivation of E.coli.

\section{ACKNOWLEDGMENTS}

The author would like to thank Fred Shaw for ordering the media and providing the equipment required for this study; Ken Keilbart for allowing access to the BCIT Food Technology Lab to undergo this study; and Bobby Sidhu, who stayed behind on multiple days to supervise the experiment, offering expert advice on conducting the study and guidance for writing this research paper. The author is also grateful to Helen Heacock and Martin MacLeod, who suggested the research topic for this study. A special note of thanks to Denny Zheng and Cathy Wong for their support and help while preparing and sharing the media and the equipment used in the BCIT Food Technology Lab.

\section{COMPETING INTEREST}

The authors declare that they have no competing interest.

\section{REFERENCES}

\footnotetext{
BBC News. (2007, January 24). Microwave 'sterilisers' warning. Retrieved from http://news.bbc.co.uk/2/hi/6293735.stm

Canadian Centre for Occupational Health and Safety [CCOHS]. (2013). Microwave ovens and their hazards. Retrieved from
} 
http://www.ccohs.ca/oshanswers/phys_agents/mic rowave_ovens.html

Centres for Disease Control and Prevention [CDC]. (2013). E. coli infection and food safety. Retrieved from http://www.cdc.gov/Features/EcoliInfection/

Donofrio, R., Bechanko, R., Hitt, N., O’Malley, K., Charnauski, T., Bestervelt, L.L., Saha, R. \& Saha, N. (2012). Are we aware of microbial hotspots in our household? Journal of Environmental Health, $75,12-19$.

Food Premises Regulation [FPR], B.C. Reg. 210/99 (1999). Retrieved from http://www.bclaws.ca/EPLibraries/bclaws_new/d ocument/ID/freeside/11_210_99

Heacock, H., Crozier, V., \& Sidhu, B. (2012a). Research methods module 5: Descriptive statistics. ENVH 8400 lecture notes (pp. 1-18), Burnaby, BC: British Columbia Institute of Technology

Heacock, H., Crozier, V., \& Sidhu, B. (2012b). Research methods module 5: Inferential statistics. ENVH 8400 lecture notes (pp. 1-17), Burnaby, BC: British Columbia Institute of Technology

Hilton, A.C. \& Austin, E. (2000). The kitchen dishcloth as a source of and vehicle for foodborne pathogens in a domestic setting. International Journal of Environmental Health Research, 10, 257-261.

Himedia. (2011). Lauryl sulphate broth (lauryl tryptose broth). Retrieved from http://himedialabs.com/TD/M080.pdf

Hygiena. (2013a). MicroSnap ${ }^{\mathrm{TM}}$ rapid microorganism detection. Retrieved from http://www.hygiena.net/docs/MicroSnap_Brochur e_RevA042013_email.pdf

Hygiena. (2013b). Micro-Snap ${ }^{\mathrm{TM}}$. Retrieved from http://www.hygiena.net/docs/Micro-Snap.pdf

Hygiena. (2013c). Directions for use of MicroSnap rapid determination of coliform and E.coli.

Hygiena. (2013d). Rapid coliform and e.coli tests awarded AOAC-RI performance tested method validation. Retrieved from http://www.hygiena.net/docs/MicroSnapAOAC_1 30722.pdf
HygienaTV. (2011, October 27). Hygiena micro-snap microbial detection: Coliforms, e.coli, total aerobic plate count. Retrieved from http://www.youtube.com/watch?v=2yvlMk_BIV0

Kitchen Sponge Safety. (n.d.). The do's and don't of sponge safety. Retrieved from http://homefoodsafety.org/vault/2499/web/files/D os $\% 20$ and $\% 20$ Donts $\% 20$ of $\% 20$ Sponge $\% 20$ Safet y.pdf

Kusumaningrum, H.D., Riboldi, G., Hazeleger, R.R, \& Beumer (2002). Survival of foodborne pathogens on stainless steel surfaces and crosscontamination to foods. International Journal of Food Microbiology, 85, 227-236.

Lee, S. (2010). Survival and growth of Escherichia coli on various commercial dish sponge/dishcloths and inhibitory effect of UV sterilization with or without moderate heat. Journal of Food Safety, 30, 721-731.

McHugh, M.L. (2013). The chi-square test of independence. Biochemia Medica, 23(2), 143149.

Microsoft Corporation. (2011a). Microsoft excel: $\operatorname{mac} 2011$.

Microsoft Corporation. (2011b). Microsoft word: $\operatorname{mac} 2011$.

NCSS 9 Statistical Software [NCSS]. (2012). Accessed, November 13, 2013. Website: www.ncss.com.

Park, D., Bitton, G. \& Melker, R. (2006). Microbial inactivation by microwave radiation in the home environment. Journal of Environmental Health, 69, 17-24.

Radiation Emitting Devices [RED] Regulations, C.R.C. c-1370 (2006). Retrieved from http://laws.justice.gc.ca/PDF/C.R.C.,_c._1370.pdf

Reagan, B.M., Rolow, A.M., \& Urban, J.E. (1982). Microwave sanitation of polyester and cotton. Textile Research Journal, 52, 186-192.

Scotch-Brite Products. (2013a). Cellulose sponge handy pack. Retrieved from http://www.scotchbrite.ca/wps/portal/3M/en_CA/CAScotchBrite/ScotchBrite/Products/Catalog/?PC_Z7_RJH9U5230GG8 80IHA65CELQCS7000000_nid=XBWHS5SCLF 
gs7XLWLFJB1Bg18K199Z16G4b1\&prodID=XB

WHS5SCLFgs\&lang=en_US

Scotch-Brite Products. (2013b). Scotch-Brite Products. Retrieved from http://www.scotchbrite.ca/wps/portal/3M/en_CA/CAScotchBrite/Scotch-Brite/Products/Catalog/

Sharma, M., Eastridge, J., \& Mudd, C. (2009). Effective household disinfection methods of kitchen sponges. Food Control, 20, 310-313.
Toronto Public Health. (2004). Food handler certification program. Retrieved from http://www.toronto.ca/health/foodhandler/pdf/fh_ document.pdf

US Food and Drug Administration [FDA]. (2013). FDA food code 2009: Chapter 4 - equipment, utensils \& linens. Retrieved from http://www.fda.gov/Food/GuidanceRegulation/Re tailFoodProtection/FoodCode/ucm188064.htm

Vollmer, M. (2004). Physics of the microwave oven. Food Physics, 39, 74-81. 